\title{
Are genetic approaches still needed to cure sickle cell disease?
}

\author{
Robert A. Brodsky' and Michael R. DeBaun ${ }^{2}$ \\ 'Division of Hematology, Department of Medicine, Johns Hopkins School of Medicine, Baltimore, Maryland, USA. ${ }^{2}$ Vanderbilt-Meharry Sickle Cell Disease Center of Excellence, \\ Vanderbilt University School of Medicine, Nashville, Tennessee, USA.
}

$\mathbf{S}$ ickle cell disease (SCD) is a heritable disorder of hemoglobin that affects 1 of every 400 black newborns and approximately 100,000 persons in the United States (1). This disease burden has a considerable impact on individuals affected and on health care systems. In the United States alone, the medical cost of caring for patients with SCD exceeds $\$ 1$ billion annually. SCD is caused by a point mutation in codon 6 of the $\beta$-globin chain that results in an amino acid substitution of valine for glutamic acid, and promotes the formation of long hemoglobin polymers under hypoxic conditions. This abnormal polymerization deforms erythrocytes and causes significant alterations in red cell integrity, rheologic properties, and lifespan. SCD leads to chronic hemolysis and a vasculopathy that involves virtually every organ. Most adults and many children develop a chronic, debilitating condition, leading to high rates of disability and unemployment. A current cohort of adults that were followed and treated with disease-modifying therapy at two large academic medical centers had a median survival of 48 years (2), which is not much different when compared with a NIH-sponsored multicenter, prospective study of a cohort of adults with SCD that was published 25 years ago (3).

Allogeneic blood or marrow transplantation (alloBMT) is the only cure for patients with sickle cell disease (SCD) (4). Worldwide, nearly 2000 children and adults with SCD have received alloBMT (5). Depending on the type of transplant and donor source, the cure rate is $90 \%-$ $95 \%$, and the risk of graft-versus-host disease (GVHD) is $4 \%-15 \%$ in the United States and Europe. Most of these data are from pediatric studies involving myeloablative conditioning regimens and stem cell grafts from matched sibling donors. Adult patients with SCD are often excluded from myeloablative BMT trials because of projected excess morbidity and mortality resulting from accumulated end-organ damage from decades of living with SCD. Additionally, many parents of children or affected individuals with SCD are reluctant to allow or receive myeloablative conditioning because of the nearly universal gonadal failure. Finding suitable donors has also been challenging. HLA-matched sibling donors are available in less than $15 \%$ of potential alloBMT recipients with SCD. Less than a quarter of African Americans have HLA matches in unrelated registries (6). Accordingly, broad application of alloBMT in SCD is dependent on novel strategies that address donor availability and limit toxicity from myeloablative conditioning regimens and GVHD. These limitations of donor availability and GVHD are driving research for novel approaches to BMT that use autologous cells with gene therapy or gene editing (Figure 1).

\section{Myeloablative gene therapy for SCD}

Gene therapy involves the harvesting of hematopoietic stem/progenitor cells (HSPCs), ex vivo transduction using a retroviral vector carrying a $\gamma$-globin or $\beta$-globin transgene, and reinfusion of transduced HSPCs following myeloablative chemotherapy. Since HSPCs are patient derived, there is no risk of GVHD; however, myeloablative chemotherapy (usually with busulfan) is required to reduce or eliminate host hematopoiesis. Myeloablative chemotherapy leads to infertility, alopecia, mucositis, and infections and may exclude patients with moderate-to-severe end-organ damage due to dose-limiting

Conflict of interest: The authors have declared that no conflict of interest exists.

Copyright: ( 2020, American Society for Clinical Investigation.

Reference information: / Clin Invest. 2020;130(1):7-9. https://doi.org/10.1172/JCI133856.

toxicities from busulfan. Stroke, a major source of morbidity, is an exclusion criterion for most gene therapy trials. There is also the potential for secondary malignancies from insertional mutagenesis and from busulfan. Self-inactivating lentiviral vectors mitigate, but do not eliminate, the risk for insertional mutagenesis. Furthermore, busulfan is seldom $100 \%$ myeloablative, and surviving HSPCs may also lead to late myeloid malignancies.

Mobilizing enough HSPCs from patients with SCD and collecting enough self-renewing HSPCs to allow life-long expression of the transgene is also a challenge. Stem cell mobilization with granulocyte CSF (G-CSF) is contraindicated in SCD; therefore, current trials are using bone marrow harvesting, which is especially painful for patients with SCD and may still result in insufficient HSPC yields for successful BMT. Plerixifor mobilization is under investigation, and early results appear promising (7). Ensuring sufficient transduction of HSPCs to allow long-term engraftment is more problematic. Lentiviral vectors can transduce self-renewing $G_{0}$ stem cells required for long-term transgene expression; however, the majority of transduced cells following peripheral blood mobilization are progenitor cells with limited to no self-renewal capacity. Progenitor cells survive three to four months and generate red cells that survive for 120 days. Moreover, autologous recovery following BMT leads to increased fetal hemoglobin that can decrease acute vaso-occlusive episodes for a year or more after BMT (8); thus, follow-up beyond two years is necessary to ensure that the transduced HSPCs are stable and sufficient to lead to long-term control of the disease.

Despite these limitations, preliminary results of gene therapy for SCD and severe $\beta$-thalassemia are encouraging, with the largest experience in severe $\beta$-thalassemia (9). In two phase I-II studies of gene therapy using a lentiviral vector and 
A
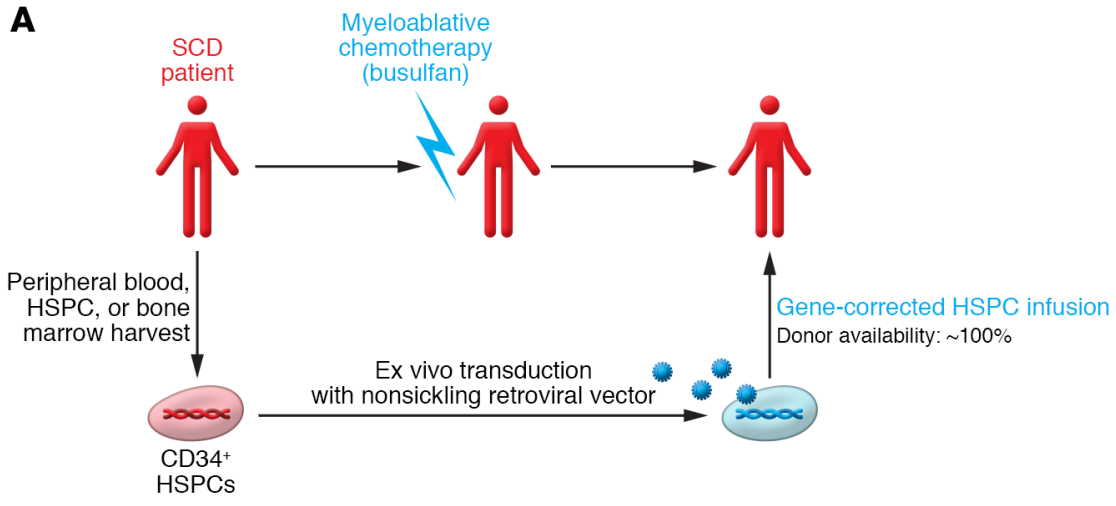

B
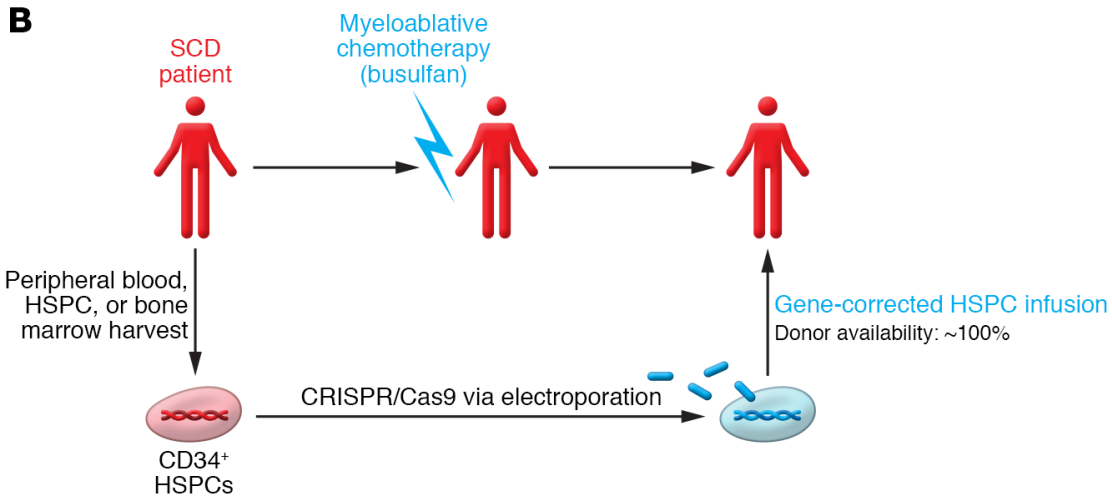

C

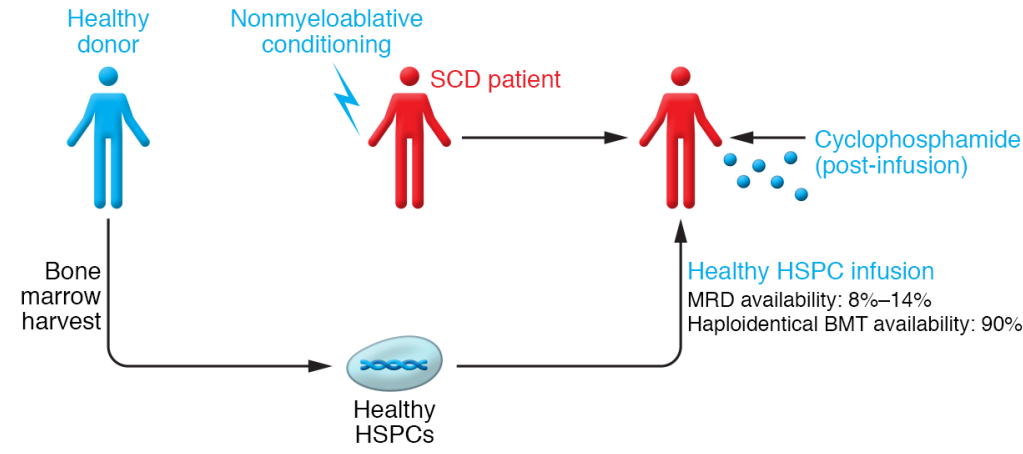

myeloablative busulfan conditioning, 12 of 13 patients with a non- $\beta^{\circ} / \beta^{0}$ genotype achieved transfusion independence, with a median follow-up of over two years. In nine patients with the $\beta^{0} / \beta^{0}$ genotype, transfusion requirements decreased, but just three of nine were able to discontinue transfusions. The first successful case report of a patient with SCD treated with gene therapy was in 2017 (10). At the time of the report, the child was 15 months from having received his transplant and no longer experiencing vaso-occlusive crises. Of note, one of two additional patients treated in the same clinical trial benefitted from the therapy. Another exciting approach to gene therapy in clinical trials is to increase production of fetal hemoglobin by knockdown of $B C L 11 A$, a gene whose product, BCL11A, regulates hemoglobin $\mathrm{F}$ expression. Reducing BCL11A thus increases the amount of nonsickling $\gamma$-globin. A number of other clinical trials involving gene therapy to treat SCD are underway. Initial data should be available within the next two to three years, but long-term data of at least five- and ten-year intervals are necessary to address late graft failure and other late effects.

\section{Myeloablative gene editing for SCD}

The approach to gene editing is similar to that for gene therapy and involves the harvesting of HSPCs, ex vivo electroporation
Figure 1. Curative approaches to SCD. (A) Gene therapy requires the harvesting of HSPCs from the patient, transduction of these cells with a nonsickling viral vector, and myeloablative chemotherapy followed by autologous BMT. (B) Gene editing also requires the harvesting of HSPCs from the patient. Gene editing of HSPCs is accomplished with electroporation of gene-editing reagents, followed by myeloablative conditioning and autologous BMT using the gene-corrected cells. (C) alloBMT can be from an HLA-matched sibling donor, a matched unrelated donor, or an HLA-haploidentical family donor. Bone marrow is harvested from a healthy donor. Traditionally, patients received myeloablative chemotherapy, but in recent years nonmyeloablative therapy, especially for HLA-haploidentical BMT with post-transplantation cyclophosphamide, has become more common. Healthy donor HSPCs are infused, followed by post-transplantation administration of cyclophosphamide to prevent GVHD and graft rejection. Children with strokes and adults with severe heart, lung, or kidney disease or strokes are typically excluded from gene therapy trials but are eligible to participate in the NIH-supported HLA-haploidentical BMT with post-transplantation cyclophosphamide phase II trial (NCT03263559). of target cells to correct the $\beta$-globin gene or to knock down BCL11A using CRISPR/ Cas9 or zinc finger nucleases, and reinfusion of genetically modified HSPCs following myeloablative chemotherapy. The toxicities and limitations from mobilization and myeloablative chemotherapy are identical to those for gene therapy protocols. No retroviral transduction is needed, but recent data on CRISPR/Cas9 editing show that the frequency of large deletions and insertions that arise near the on-target site is higher than originally thought (11). Moreover, since DNA breaks induce apoptosis in healthy cells, it appears that there is enrichment of edited HSPCs with deficient p53, raising additional safety concerns regarding cancer risk (12). 


\section{Nonmyeloablative haploidentical BMT for SCD}

The approach to nonmyeloablative haploidentical BMT was developed to increase donor availability and to provide curative options for adults with SCD who have preexisting heart, lung, and kidney dysfunction that would preclude myeloablative therapy. For children and adults with SCD, multiple previous unsuccessful single-center nonmyeloablative, haploidentical BMT protocols were initiated and abandoned because of transplant-related mortality. However, the more recent generation of nonmyeloablative, HLA-haploidentical BMT with post-transplantation cyclophosphamide, roughly one-third the cost of myeloablative gene therapy and gene editing, has dramatically improved the clinical outcome of children and adults with SCD.

Virtually every patient eligible for a gene therapy or gene editing trial is also eligible for an HLA-haploidentical BMT with post-transplantation cyclophosphamide. Transplantation trials are more inclusive in that most gene therapy trials exclude patients who have had a stroke. The first clinical trial of nonmyeloablative, HLA-haploidentical BMT with post-transplantation cyclophosphamide for SCD in 2012 reported a graft failure rate of approximately $40 \%$ (13); however, subsequent modifications to the preparative regimens involving the addition of thiotepa or an increase in the dose of total body irradiation from 200 cGy to 400 cGy increased engraftment to $90 \%$ without adding to toxicity (14-16). The collective results from these three recent studies ( $n=39$ patients with SCD) showed no mortality, an engraftment rate of $90 \%$, and a rate of GVHD above grade 2 of $8 \%$. A clinical trial sponsored by the National Heart, Lung, and Blood Institute (NHLBI) involving HLA-haploidentical BMT with post-transplantation cyclophosphamide for SCD at more than 30 clinical centers throughout the United States and Europe is currently underway (NCT03263559). Confirmation of these encouraging early results will confirm that myeloablative conditioning and full-matched HLA donors are no longer necessary to cure SCD.

\section{Are genetic approaches still necessary to cure SCD?}

The era of curative therapy for patients with SCD is upon us. NIH-sponsored nonmyeloablative, HLA-haploidentical BMT with post-transplantation cyclophosphamide offers the opportunity to cure up to $95 \%$ of the children and $90 \%$ of the adults with severe SCD. Clinical trials involving myeloablative gene therapy and genome editing are also underway with 100\% donor availability but are limited predominantly to children who can tolerate the myeloablative regimen. Although randomized, controlled trials comparing the two strategies are not likely to be undertaken, understandably, curative therapies that include nonmyeloablative methods will commonly be selected over those that are myeloablative.

Informed families with SCD have multiple options to enroll in clinical trials designed to cure and advance care for the next generation. The pressing challenges are to include full disclosure of the various curative options for children and adults with SCD, to minimize late effects from preparative regimens, and to advance innovative science leading to nonmyeloablative, haploidentical BMT, gene therapy, or gene-editing trials. The future for curing children and adults with SCD looks bright.

Address correspondence to: Robert A. Brodsky, 720 Rutland Avenue, Ross Room 1025, Baltimore, Maryland 21205, USA. Phone: 410.502.2546; Email: brodsro@ jhmi.edu.

1. Kato GJ, et al. Sickle cell disease. Nat Rev Dis Primers. 2018;4:18010.

2. DeBaun MR, et al. Decreased median survival of adults with sickle cell disease after adjusting for left truncation bias: a pooled analysis. Blood. 2019;133(6):615-617.

3. Platt OS, et al. Mortality in sickle cell disease.
Life expectancy and risk factors for early death. N Engl J Med.1994;330(23):1639-1644.

4. Bolaños-Meade J, Brodsky RA. Blood and marrow transplantation for sickle cell disease: is less more? Blood Rev. 2014;28(6):243-248.

5. Gluckman E, et al. Sickle cell disease: an international survey of results of HLA-identical sibling hematopoietic stem cell transplantation. Blood. 2017;129(11):1548-1556

6. Justus D, Perez-Albuerne E, Dioguardi J, Jacobsohn D, Abraham A. Allogeneic donor availability for hematopoietic stem cell transplantation in children with sickle cell disease. Pediatr Blood Cancer. 2015;62(7):1285-1287.

7. Esrick EB, et al. Successful hematopoietic stem cell mobilization and apheresis collection using plerixafor alone in sickle cell patients. Blood $A d v$. 2018;2(19):2505-2512.

8. Ferster A, et al. Transplanted sickle-cell disease patients with autologous bone marrow recovery after graft failure develop increased levels of fetal haemoglobin which corrects disease severity. Br J Haematol. 1995;90(4):804-808.

9. Thompson AA, et al. Gene Therapy in Patients with Transfusion-Dependent $\beta$-Thalassemia. NEngl J Med. 2018;378(16):1479-1493.

10. Ribeil JA, et al. Gene therapy in a patient with sickle cell disease. $N$ Engl J Med. 2017;376(9):848-855.

11. Kosicki M, Tomberg K, Bradley A. Repair of double-strand breaks induced by CRISPR-Cas 9 leads to large deletions and complex rearrangements. Nat Biotechnol. 2018;36(8):765-771.

12. Haapaniemi E, Botla S, Persson J, Schmierer B, Taipale J. CRISPR-Cas9 genome editing induces a p53-mediated DNA damage response. Nat Med. 2018;24(7):927-930.

13. Bolaños-Meade J, et al. HLA-haploidentical bone marrow transplantation with posttransplant cyclophosphamide expands the donor pool for patients with sickle cell disease. Blood. 2012;120(22):4285-4291.

14. Fitzhugh CD, et al. Cyclophosphamide improves engraftment in patients with SCD and severe organ damage who undergo haploidentical PBSCT. Blood Adv. 2017;1(11):652-661.

15. de la Fuente J, et al. Haploidentical bone marrow transplantation with post-transplantation cyclophosphamide plus thiotepa improves donor engraftment in patients with sickle cell anemia: results of an international learning collaborative. Biol Blood Marrow Transplant. 2019;25(6):1197-1209.

16. Bolaños-Meade J, et al. Effect of increased dose of total body irradiation on graft failure associated with HLA-haploidentical transplantation in patients with severe haemoglobinopathies: a prospective clinical trial. Lancet Haematol. 2019;6(4):e183-e193. 